\title{
Células inflamatórias infiltrantes: Perfil e distribuição em carcinomas mamários de
}

\author{
gatas \\ Infilltrating inflammatory cells: Profile and distribution in feline mammary carcinomas \\ Células inflamatorias infiltrantes: Perfil y distribución en carcinomas mamarios de gatos
}

Recebido: 08/03/2021 | Revisado: 15/03/2021 | Aceito: 20/03/2021 | Publicado: 27/03/2021

Michele Berselli

ORCID: https://orcid.org/0000-0002-0563-4139 Universidade de Santa Cruz do Sul, Brasil E-mail: michele.berselli@gmail.com

Thomas Normanton Guim

ORCID: https://orcid.org/0000-0001-8278-1397 Universidade Federal de Pelotas, Brasil

E-mail: thomasguim@ hotmail.com

Clarissa Caetano de Castro

ORCID: https://orcid.org/0000-0002-4822-034X Universidade Federal de Pelotas, Brasil E-mail: clarissac.decastro@gmail.com

Luísa Grecco Corrêa

ORCID: https://orcid.org/0000-0003-1355-2907 Universidade Federal de Pelotas, Brasil

E-mail: luisagcorrea@gmail.com

Andressa Dutra Piovesan Rossato

ORCID: https://orcid.org/0000-0001-5760-9392 Instituto de Desenvolvimento Educacional do Alto Uruguai, Brasil E-mail: andressa-piovesan@ @otmail.com

Luísa Mariano Cerqueira da Silva

ORCID: https://orcid.org/0000-0001-6158-4204 E-mail: luisamarianovet@yahoo.com.br

Fabiane Borelli Grecco

ORCID: https://orcid.org/0000-0002-3996-315X Universidade Federal de Pelotas, Brasil E-mail: fabianegrecco18@gmail.com

Fabio Raphael Pascoti Bruhn

ORCID: https://orcid.org/0000-0002-4191-965X Universidade Federal de Pelotas, Brasil

E-mail: fabio_rpb@yahoo.com.br

Cristina Gevehr Fernandes

ORCID: https://orcid.org/0000-0002-0729-2099 Universidade Federal de Pelotas, Brasil

E-mail: crisgevf@yahoo.com.br

\begin{abstract}
Resumo
Tumores mamários malignos têm um número relativamente alto de linfócitos T infiltrantes. Atualmente, os linfócitos $\mathrm{B}$ infiltrados em tumores têm sido reconhecidos como um potencial marcador no câncer de mama humano. Este estudo teve como objetivo relacionar fatores prognósticos de tumores mamários ao padrão de infiltrado inflamatório em gatas. Foram analisados protocolos de 38 animais diagnosticados com tumor mamário e obtido dados sobre sexo, idade e raça. As amostras foram avaliadas quanto a distribuição, tipo histológico, tamanho, grau histológico, metástase linfonodal, distribuição celular inflamatória e intensidade da inflamação. Para marcação imunoistoquímica, foi utilizado anticorpo monoclonal anti-CD79a. Houve predomínio de gatas idosas e sem raça definida. Carcinomas tubulares, sólidos e cribriformes foram os mais diagnosticados. Tumores de grau III menores que 2,0 cm foram frequentes. O infiltrado inflamatório total era predominantemente multifocal e de baixa contagem, independentemente do tipo histológico, grau e tamanho. Os linfócitos foram as células mais frequentes e de baixa contagem. A imunocoloração CD79a foi observada na maioria dos neoplasmas, mostrando que linfócitos B e plasmócitos são componentes integrais do infiltrado inflamatório do tumor e de distribuição predominantemente difusa. Células CD79a positivas apresentaram diferenças significativas para distribuição $(p=0,038)$, no tamanho $(p=0,045)$ e invasão linfática $(\mathrm{p}=0,039)$. Este é o primeiro estudo a quantificar as células inflamatórias do microambiente tumoral mamário de gatas e revela resultados iniciais promissores. As células CD79a positivas constituem uma parcela significativa da população linfocitária do microambiente tumoral e fazem parte da resposta inflamatória associada ao tumor em carcinomas mamários felinos.
\end{abstract}


Palavras-chave: Felino; Infiltrado inflamatório; Linfócito; Tumor mamário.

\begin{abstract}
Malignant mammary tumors have a relatively high number of infiltrating T lymphocytes. Currently, B lymphocytes infiltrated in tumors have been recognized as a potential marker in human breast cancer. The aim of this study was to relate prognostic factors of mammary tumors to the pattern of inflammatory infiltrate in cats. Protocols of 38 animals diagnosed with mammary tumor were analyzed and data on sex, age and race were obtained. The samples were evaluated for distribution, histological type, size, histological grade, lymph node metastasis, inflammatory cell distribution and intensity of inflammation. For immunohistochemical staining, anti-CD79a monoclonal antibody was used. There was a predominance of elderly and mixed breed cats. Tubular, solid and cribriform carcinomas were the most diagnosed. Grade III tumors smaller than $2.0 \mathrm{~cm}$ were frequent. The total inflammatory infiltrate was predominantly multifocal and of low count, regardless of the histological type, degree and size. Lymphocytes were the most frequent and low-count cells. CD79a immunostaining was observed in most neoplasms, showing that B lymphocytes and plasma cells are integral components of the inflammatory infiltrate of the tumor and of predominantly diffuse distribution. Positive CD79a cells showed significant differences in distribution $(p=0.038)$, in size $(\mathrm{p}=0.045)$ and lymphatic invasion $(\mathrm{p}=0.039)$. This is the first study to quantify inflammatory cells in the mammary tumor microenvironment of cats and reveals promising initial results. CD79a positive cells constitute a significant portion of the lymphocyte population in the tumor microenvironment and are part of the tumor-associated inflammatory response in feline breast carcinomas.
\end{abstract}

Keywords: Feline; Inflammatory infiltrate; Lymphocyte; Mammary tumor.

\begin{abstract}
Resumen
Los tumores de mama malignos tienen un número relativamente alto de linfocitos $\mathrm{T}$ infiltrantes. Actualmente, los linfocitos B infiltrados en tumores se han reconocido como un marcador potencial en el cáncer de mama humano. Este estudio tuvo como objetivo relacionar los factores pronósticos de los tumores de mama con el patrón de infiltrado inflamatorio en gatos. Se analizaron protocolos de 38 animales diagnosticados con tumor de mama y se obtuvieron datos sobre sexo, edad y raza. Las muestras se evaluaron en cuanto a distribución, tipo histológico, tamaño, grado histológico, metástasis ganglionar, distribución de células inflamatorias e intensidad de la inflamación. Para la tinción inmunohistoquímica, se utilizó anticuerpo monoclonal anti-CD79a. Hubo un predominio de gatos ancianos y mestizos. Los carcinomas tubulares, sólidos y cribiformes fueron los más diagnosticados. Fueron frecuentes los tumores de grado III menores de 2,0 cm. El infiltrado inflamatorio total fue predominantemente multifocal y de bajo recuento, independientemente del tipo histológico, grado y tamaño. Los linfocitos fueron las células más frecuentes y de menor recuento. Se observó inmunotinción de CD79a en la mayoría de las neoplasias, lo que demuestra que los linfocitos B y las células plasmáticas son componentes integrales del infiltrado inflamatorio del tumor y de distribución predominantemente difusa. Las células CD79a positivas mostraron diferencias significativas en la distribución $(\mathrm{p}=0.038)$, en tamaño $(\mathrm{p}=0.045)$ e invasión linfática $(\mathrm{p}=0.039)$. Este es el primer estudio que cuantifica las células inflamatorias en el microambiente del tumor mamario de los gatos y revela resultados iniciales prometedores. Las células CD79a positivas constituyen una porción significativa de la población de linfocitos en el microambiente tumoral y son parte de la respuesta inflamatoria asociada al tumor en los carcinomas de mama felinos.
\end{abstract}

Palabras clave: Felino; Infiltrado inflamatório; Linfocito; Tumor mamario.

\title{
1. Introdução
}

As neoplasias mamárias são descritas como o terceiro tipo mais frequente de tumor em felinos cujo prognóstico varia de reservado a ruim, devido sua agressividade (Cassali et al., 2018; Cassali et al., 2020). Vários parâmetros são considerados importantes indicadores prognósticos para estes neoplasmas, tais como: idade, tamanho primário do tumor, comprometimento de linfonodos, número de mitoses, extensão da necrose, margens cirúrgicas e tipo de invasão (Goldschmidt, Peña \& Zappulli, 2017; Zappulli et al., 2015). Além disso, outro fator que pode estar envolvido na progressão tumoral é a resposta inflamatória associada ao tumor (Carvalho et al., 2011; Franzoni et al., 2019; O’Neill et al., 2009).

Os tumores mamários malignos são frequentemente associados a um número relativamente alto de linfócitos infiltrantes (Carvalho et al., 2016). O significado prognóstico do infiltrado de linfócitos $\mathrm{T}$ em tumores tem sido amplamente aceito em neoplasmas humanos e caninos, uma vez que cães com alto nível de infiltrado inflamatório e linfócitos T nos tecidos tumorais apresentaram menor tempo de sobrevivência (Carvalho et al., 2016; Franzoni et al., 2019; Lopes-Neto et al., 2017; Wouters \& Nelson, 2018). Dados a respeito do infiltrado de linfócitos em neoplasmas de felinos são escassos. Em cães, sabe-se que os tumores mamários malignos são caracterizados por infiltração acentuada de linfócitos, não sendo observados linfócitos 
no tecido mamário normal e em tumores benignos, o que sugere que linfócitos, principalmente os linfócitos T, desempenham um papel importante tanto na progressão como na supressão tumoral. Além disso, um número expressivo de células B está associado a um comportamento tumoral agressivo em cães e os linfócitos B se infiltraram mais profundamente no tecido do que as células T (Kim,Yu, Yhee, Im \& Sur, 2010; Kim, Chon, Im, Kim, \& Sur, 2013; Lopes-Neto et al., 2017; Souza et al., 2018).

Atualmente os linfócitos B infiltrantes em tumores têm sido reconhecidos como um potencial marcador em câncer de mama humano (Shen,Wang \& Ren, 2018). As células B desempenham um papel importante na modulação da resposta imune ao câncer e são a segunda população de linfócitos infiltrantes de tumor mais abundante (Yuen, Demissie \& Pillai, 2016). As células B no câncer humano podem desempenhar papeis distintos, podendo inibir o desenvolvimento do tumor pela produção de anticorpos, ativação de células NK, estimular a fagocitose por macrófagos e ainda pela ativação de células CD4+ e CD8+, podendo representar uma resposta de anticorpos contra antígenos nos tumores da mama em humanos ou ao contrário, podem promover o desenvolvimento tumoral suprimindo as respostas das células T citotóxicas (Mahmoud et al., 2012; Yuen et al., 2016).

Em gatos, é provável que linfócitos $\mathrm{T}$, células $\mathrm{B}$ e plasmócitos possam participar da regulação negativa do crescimento tumoral em carcinomas de células escamosas (Pérez, Day, Martín, González \& Mozos, 1999). Dados a respeito do papel dos linfócitos em tumores mamários de felinos não são conhecidos, portanto, o objetivo deste trabalho foi analisar e descrever o perfil de distribuição de linfócitos, e realizar a análise imunoistoquímica dos linfócitos B e plasmócitos (CD79a positivos) no infiltrado inflamatório dos neoplasmas mamários de felinos e determinar se as características desse infiltrado estavam relacionadas com o comportamento biológico dos tumores.

\section{Metodologia}

\subsection{Levantamento de dados}

Foi realizado um estudo retrospectivo referente aos tumores mamários de gatas do Serviço de Oncologia Veterinária/ de Pelotas (SOVET/ UFPEL) desde 2014, do qual resultaram em dados de 38 gatas totalizando 77 casos de neoplasmas mamários. Foram tabuladas as seguintes informações: idade, raça, diagnósticos morfológico e histológico, número de tumores, tamanho e grau histológico e avaliação das células inflamatórias associadas ao tumor (distribuição das células inflamatórias, intensidade do infiltrado inflamatório total e intensidade do infiltrado inflamatório linfocitário).

\subsection{Classificação das amostras tumorais}

Foi realizado incialmente o levantamento dos neoplasmas mamários. Os tumores foram classificados como únicos, múltiplos e multifocais. Foram considerados multifocais aqueles que apresentaram nódulos do mesmo tipo histológico e múltiplos aqueles que apresentaram neoplasmas de diferentes tipos histológicos, podendo ter combinações de diferentes tumores malignos ou combinações de benignos e malignos.

As amostras foram classificadas histologicamente conforme preconizado pela literatura (Cassali et al., 2020; Goldschmidt et al., 2017). Foram incluídas no estudo amostras classificadas como carcinomas mamários, considerando-se os tipos histológicos comuns da glândula mamária de gatas (Cassali et al., 2020; Goldschmidt et al., 2017).

O grau histológico foi definido de acordo com Elston e Ellis (1991), e se baseia no somatório dos escores de três parâmetros: formação tubular, pleomorfismo celular e índice mitótico. O tamanho dos tumores foi definido através da mensuração em centímetros da massa tumoral em três dimensões (Comprimento x Largura x Altura), sendo considerado para avaliação o maior valor. Os neoplasmas foram classificados através dos valores de tamanho para tumores mamários em felinos onde T1 é atribuído a neoplasmas de 0 a $<2 \mathrm{~cm}, \mathrm{~T} 2$ corresponde a tumores medindo 2 a $3 \mathrm{~cm}$ e T3 corresponde a tumores com 
mais de $3 \mathrm{~cm}$ (Cassali et al., 2020).

\subsection{Avaliação do Infiltrado inflamatório}

A avaliação das células inflamatórias associadas ao tumor nos carcinomas mamários foi realizada levando em consideração três parâmetros distintos: distribuição das células inflamatórias, intensidade do infiltrado inflamatório total e intensidade do infiltrado inflamatório linfocitário. A distribuição do infiltrado inflamatório total nos carcinomas mais frequentes (tubular, sólido e cribriforme), foi classificada nas áreas periféricas e intratumorais em: i) focal: presença de 1-3 focos inflamatórios; ii) multifocal: presença de mais de três focos inflamatórios e iii) difusa: presença de células inflamatórias uniformemente distribuídas na secção de tumor (Estrela-Lima et al., 2010; Lopes-Neto et al., 2017).

A intensidade do infiltrado inflamatório total e a intensidade linfocitária foi analisado nos carcinomas tubulares, cribriformes e sólidos de gatas com tumores únicos e multifocais. Estes foram categorizados em dois subgrupos cada um: baixa contagem (<800 linfócitos) e alta contagem ( $>800$ linfócitos), com base na análise do infiltrado inflamatório (Estrela-Lima et al., 2010; Lopes-Neto et al., 2017).

Os dados acerca dos parâmetros de inflamação foram obtidos com a avaliação de cinco campos mais representativos ("hot spots") em cortes histológicos de $4 \mu \mathrm{m}$ coradas pelo Hematoxilina Eosina (HE) em aumento de 400x. Para tal, foi realizada a contagem das células inflamatórias em imagens capturadas em câmera digital adaptada a um Microscópio Olympus CX-21.

\subsection{Imunoistoquímica}

A imunomarcação para CD79a foi realizada para detecção de linfócitos B e plasmócitos em cortes desparafinados de $3 \mu \mathrm{m}$ em lâminas positivadas. Realizou-se o bloqueio da peroxidase endógena em $\mathrm{H} 2 \mathrm{O} 2$ a 3\%. A recuperação antigênica foi realizada em micro-ondas em solução de TRIS EDTA. Posteriormente os cortes foram tratados com solução de leite em pó desnatado 5\% para o bloqueio das ligações inespecíficas (background). Foi utilizado o anticorpo CD79a monoclonal (CM067A - BIOCARE $^{\circledR}$ ) na diluição 1:150 e os cortes foram incubados em câmara úmida por 12-14 horas (overnight) a $4{ }^{\circ} \mathrm{C}$. Após a incubação, utilizou-se o sistema de detecção por peroxidase (HRP) utilizando o kit Mouse-on-Canine (Promark - BIOCARE ${ }^{\circledR}$ ), por 60 minutos. Posteriormente, a cor foi revelada com tetrahidrocloreto de 3,3'-diaminobenzidina (DAB) a 0,05\% com $1 \mathrm{ml}$ de tampão e as lâminas foram contra-coradas com hematoxilina. $\mathrm{O}$ anticorpo primário foi substituído por PBS nos controles negativos e controles positivos consistiram de seções de linfonodos.

As amostras foram avaliadas semiquantitativamente, sendo o infiltrado categorizado como: Ausente - sem células B ou plasmócitos detectáveis; Difuso - células B ou plasmócitos espalhados nos focos de infiltrado inflamatório por todo o tumor; Agregados - células organizadas em folículos e/ou agregados densos, baseado nos critérios descritos por Knief et al. (2016). A presença de células CD79a+ foi também avaliada quanto o padrão de distribuição no estroma tumoral em focal, multifocal e difuso. Além disso, as amostras marcadas por imunoistoquímica foram avaliadas quantitativamente, através da contagem sequencial em 10 campos aleatórios, não adjacentes, em objetiva de 400x, obtendo-se uma média da contagem de células CD79a+, conforme metodologia adaptada de Estrela-Lima (2010) e Knief et al., (2016).

\subsection{Critérios de inclusão/exclusão de amostras}

Para o levantamento de dados epidemiológicos, morfológicos e de localização dos neoplasmas, todas as amostras foram incluídas na análise. Para a avaliação do grau, tamanho, distribuição e média de células inflamatórias e linfocitárias, foram incluídos todos os neoplasmas diagnosticados como carcinomas tubulares sólidos e cribriformes por serem os tumores mais frequentemente diagnosticados nas gatas. Foram censurados tumores benignos e alterações não neoplásicas. 
Para a análise da intensidade do infiltrado inflamatório, linfocitário e imunoistoquímica, foram incluídas na análise apenas os neoplasmas de animais com tumores únicos e multifocais, e os tipos histológicos mais frequentes: carcinomas tubulares, sólidos e cribriformes. Foram censurados os nódulos de gatas com neoplasmas múltiplos e os que apresentaram recidivas de tipos histológicos diferentes, devido à variabilidade de tipos histológicos no mesmo animal.

\subsection{Estatística}

A categorização dos neoplasmas mamários quanto a raça, idade, comportamento biológico, tipo histológico, grau, tamanho, distribuição e intensidade do infiltrado inflamatório total e infiltrado linfocitário foram expressas como porcentagem. Para a análise quantitativa dos dados, o teste de Kolmogorov-Smirnov foi inicialmente utilizado para verificar a normalidade da distribuição.

O infiltrado inflamatório total e linfocitário foi expresso como média \pm desvio padrão. A correlação entre o infiltrado inflamatório e o grau de tumor, tamanho e tipo histológico foi analisada utilizando o teste não paramétrico de correlação de Spearman. Para a análise qualitativa dos dados, foram utilizados dois intervalos de intensidade de infiltrado inflamatório total e linfocitário baixa contagem ( $<800$ linfócitos) e alta contagem ( $>800$ linfócitos).

Foram utilizados métodos não paramétricos para descrição e teste das células CD79a+, dado que o número de amostras serem menor que 30 e não seguirem distribuição normal. Os dados numéricos quanto ao infiltrado das células CD79a + foram descritos como média \pm desvio padrão. Os escores das células CD79a positivas foram expressos como porcentagem. As correlações de classificação de Spearman foram utilizadas para descrever as associações entre o resultado da imunoistoquímica e os fatores prognósticos analisados. As diferenças entre os grupos foram avaliadas pelo teste U de MannWhitney (quando dois grupos foram comparados) ou Kruskal-Wallis (mais de dois grupos).

$\mathrm{O}$ teste de qui-quadrado de Pearson foi utilizado para avaliar a associação entre as variáveis categóricas. A significância estatística foi considerada quando $\mathrm{P}<0,05$ e as análises foram realizadas no software IBM SPSS22 ${ }^{\circledR}$.

\section{Resultados e Discussão}

Foram avaliadas 38 gatas, das quais 92,1\% (35/38) eram sem raça definida, 5,3\% (2/38) eram siameses e um não teve a raça informada. O predomínio de gatas sem raça definida reflete a casuística maior de amostras provenientes de animais sem raça definida recebidos pelo SOVET-LRD/UFPEL. Felinos da raça siamesa tem maior predisposição ao desenvolvimento de neoplasmas mamários (Zappulli et al., 2015; Saif et al., 2016), embora no Brasil a predisposição racial não tenha sido ainda observada (Cunha et al., 2017; Cassali et al., 2018). A idade variou de 6 a 21 anos, com idade média de 11,8 anos para o aparecimento de neoplasmas mamários. Esses tumores são mais frequentes em animais idosos (Togni, Masuda, Kommers, Fighera \& Irigoyen, 2013; Cassali et al., 2018).

Da avaliação de amostras de 38 animais, resultaram 77 diagnósticos de neoplasmas mamários, devido ao fato que 55,3\% (21/38) dos animais apresentaram mais de um nódulo. Este dado difere do encontrado por Borrego, Cartagena \& Engel (2009) onde 87\% dos neoplasmas encontrados em 23 gatas eram solitários. Dos animais com mais de um nódulo, 36,8\% (14/38) apresentaram tumores múltiplos e 18,5\% (7/38) tumores multifocais. Apenas. 44,7\% (17/38) dos animais apresentaram nódulos únicos. Destes, 97,4\% (75/77) eram neoplasmas malignos.

Quanto aos tipos histológicos malignos os mais frequentes foram os carcinomas tubulares (37,3\% ([28/75]), os carcinomas sólidos (32\% ([24/75]), os carcinomas cribriformes (22,7\% ([17/75]), e os carcinomas papilares (4\% ([3/75]). O carcinoma lobular, o carcinoma de células escamosas e o adenomioepitelioma maligno foram os menos frequentes e representaram 1,3\% (1/75) dos casos cada um. Sabe-se que os tipos histológicos encontrados podem ser diversificados, os carcinomas tubulares, papilares, sólidos, cribriformes e in situ são considerados diagnósticos comuns na glândula mamária de 
gatas (Campos et al., 2016; Cassali et al., 2018).

Para a avaliação do grau, tamanho e distribuição do infiltrado inflamatório, foram analisados 66 neoplasmas com diagnóstico de carcinomas tubulares, cribriformes e sólidos (Figura 1). Em relação ao grau, 59,1\% (39/66) dos tumores malignos eram grau III, 31,8\% (21/66) grau II e 7,6\% (5/66) grau I, não sendo possível avaliar o grau de apenas um neoplasma. Neoplasmas mamários de grau II e III são frequentes em felinos (Borrego et al., 2009; Zappulli et al., 2015; De Campos et al., 2016; Seixas, Antunes, Pires, 2018). O grau histológico influencia significativamente o comportamento biológico dos tumores mamários felinos, principalmente os de grau III e seu efeito se sobrepõe a outros fatores, como idade, tamanho do tumor e tipo histológico (Seixas, Palmeira, Pires, Bento \& Lopes, 2011). Portanto, fatores prognósticos adicionais são necessários para diferenciar o seu comportamento biológico (Viste, Myers, Singh \& Simko, 2002).

Figura 1 - Tipos histológicos de carcinoma mamário de gatas (Hematoxilina-eosina) 40X. A) Carcinoma tubular composto por proliferação epitelial disposta de forma predominantemente tubular. B) Carcinoma sólido constituído por células epiteliais que formam cordões ou ninhos de células, sem formação de luz tubular. C) Carcinoma cribriforme composto por proliferação de células epiteliais neoplásicas formando um arranjo semelhante a uma peneira.

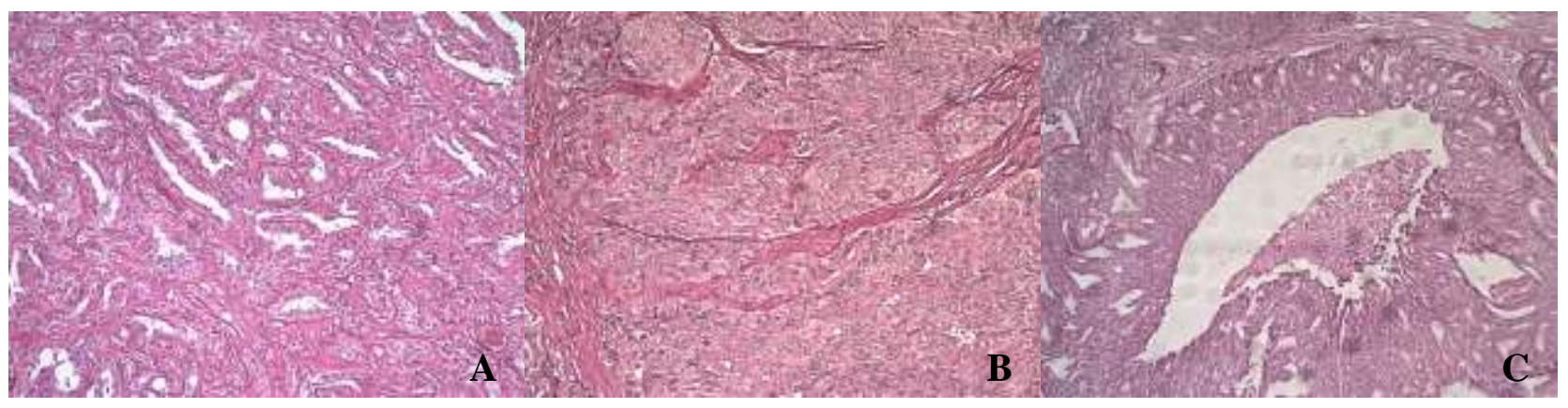

Fonte: Autores.

Quanto o tamanho dos neoplasmas malignos, 51,5\% (34/66) eram de tamanho T1; 16,7\% (11/66) de tamanho T2 e $21,2 \%$ (14/66) de tamanho T3. Não foram informados os tamanhos de 10,6\% (7/66) dos neoplasmas, pois se tratavam de fragmentos, sem informação do tamanho total. Neste estudo, a frequência de tumores de tamanho menor que $2 \mathrm{~cm}$ foi elevada, fato que poderia estar relacionado a um melhor prognóstico, já que o tamanho é um importante fator prognóstico na espécie felina (Viste et al., 2002; Zappulli et al., 2015). No entanto, neste estudo, verificamos que a maioria dos tumores T1 eram grau III.

A distribuição do infiltrado inflamatório total nos neoplasmas foi predominantemente multifocal em 84,8\% (56/66) dos casos, tanto nas regiões peritumorais como intra-tumorais, não diferindo com o encontrado na literatura em humanos e caninos (Macchetti et al., 2006; Estrela-Lima et al., 2010; Salgado et al., 2015; Carvalho et al., 2016). Apenas 10,7\% (7/66) apresentaram infiltrado inflamatório difuso e 4,5\% (3/66) infiltrado inflamatório focal. Não houve diferenças significativas no padrão de distribuição entre as categorias tipo histológico, grau e tamanho. Não foi possível realizar a contagem de células inflamatórias em três neoplasmas por não atenderem os critérios de contagem.

A média de células inflamatórias totais nos 66 neoplasmas foi de $835 \pm 654$, com predomínio de linfócitos na quase totalidade dos neoplasmas onde a média foi de $791 \pm 659$. Plasmócitos e macrófagos foram encontrados em número reduzido na maioria dos neoplasmas em relação ao número de linfócitos (Figura 2). Em apenas um caso o infiltrado era predominantemente histiocítico e neutrofílico. Tem sido relatado que populações heterogêneas das células do sistema imune frequentemente se infiltram nos tumores mamários, cujos linfócitos são as principais células em neoplasmas mamários de caninos (Lopes-Neto et al., 2017; de Souza et al., 2018) não diferindo ao encontrado nas amostras de felinos. 
Figura 2 - Composição do infiltrado inflamatório nos carcinomas mamários felinos.

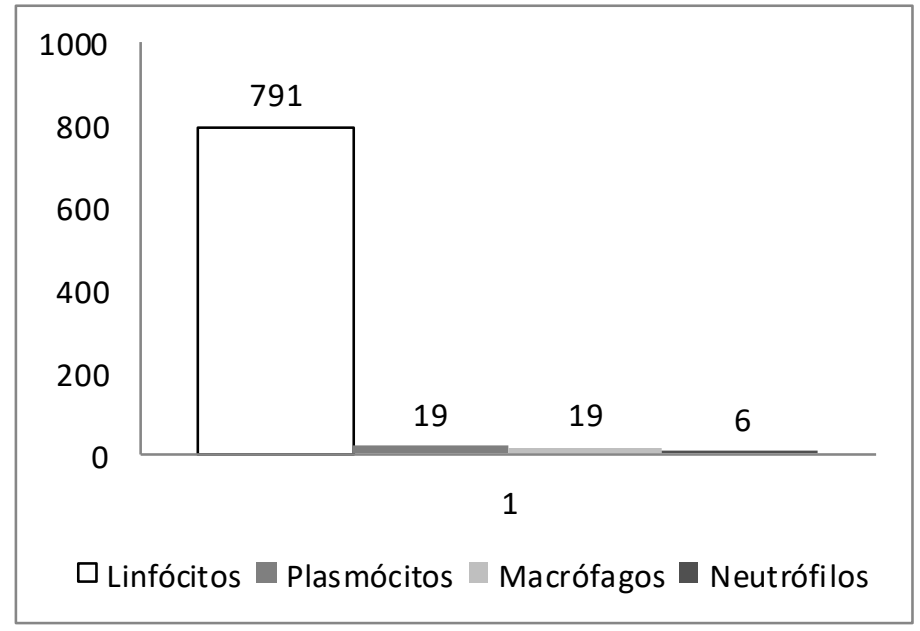

Fonte: Autores.

Neste estudo, a média das células inflamatórias totais e linfocitárias dos carcinomas tubulares, sólidos e cribriformes quanto aos paramentos tipo histológico, grau e tamanho, invasão tecidual e linfática foi avaliado em 33 tumores (Tabela 1). Gatas com neoplasmas de mais de um tipo histológico diferente foi censurada, pois alguns dos tumores pertenciam a mesma mama ou a glândulas mamárias próximas, desta forma, poderia haver interferência no número e intensidade do infiltrado inflamatório.

Tabela 1 - Quantificação das células inflamatórias totais e linfocitárias nos carcinomas mamários de gatas.

\begin{tabular}{|c|c|c|c|c|c|c|}
\hline \multirow{2}{*}{ Parâmetros } & & \multirow{2}{*}{$\mathbf{N}$} & \multicolumn{2}{|c|}{ Infiltrado total } & \multicolumn{2}{|c|}{ Infiltrado linfocitário } \\
\hline & & & Média & $\mathbf{D P}$ & Média & $\mathbf{D P}$ \\
\hline \multirow{3}{*}{$\begin{array}{l}\text { Grau } \\
\text { histológico }\end{array}$} & Grau I & 4 & 525,10 & 339,91 & 517,85 & 334,29 \\
\hline & Grau II & 7 & 886,86 & 481,07 & 860,80 & 481,45 \\
\hline & Grau III & 21 & 885,30 & 711,52 & 850,46 & 705,05 \\
\hline \multirow{3}{*}{$\begin{array}{l}\text { Tipo } \\
\text { histológico }\end{array}$} & Carcinoma Tubular & 13 & 649,40 & 323,09 & 625,65 & 324,35 \\
\hline & Carcinoma cribriforme & 8 & 895,75 & 470,34 & 855,85 & 466,40 \\
\hline & Carcinoma Sólido & 12 & 986,88 & 896,54 & 958,62 & 885,89 \\
\hline \multirow{3}{*}{ Tamanho } & $\mathrm{T} 1(<2 \mathrm{~cm})$ & 19 & 901,59 & 694,40 & 876,51 & 695,05 \\
\hline & $\mathrm{T} 2(2-3 \mathrm{~cm})$ & 5 & 647,80 & 411,85 & 606,92 & 415,04 \\
\hline & $\mathrm{T} 3(>3 \mathrm{~cm})$ & 5 & 488,60 & 101,57 & 473,92 & 93,16 \\
\hline \multirow{2}{*}{$\begin{array}{l}\text { Invasão } \\
\text { tecidual }\end{array}$} & Sim & 23 & 874,02 & 597,41 & 848,69 & 598,92 \\
\hline & Não & 9 & 755,27 & 739,29 & 715,2 & 711,94 \\
\hline Invasão & Sim & 13 & 895,45 & 847,92 & 861,48 & 837,97 \\
\hline linfática & Não & 19 & 803,11 & 448,57 & 776,71 & 445,97 \\
\hline
\end{tabular}

Fonte: Autores.

Verificamos que a média das células inflamatórias totais e linfocitárias foi maior nos tumores T1 em relação aos tumores T3, assim como nos Carcinomas sólidos em relação aos tubulares e nos tumores grau III em relação ao grau I (Tabela 1). Em um estudo em caninos, foi demostrado que houve uma associação entre o número de células CD3 + e o grau do tumor, onde neoplasmas graus II e III apresentaram maior número de células CD3+ do que em outros tumores, além disso, os cães com um alto nível de infiltrado inflamatório nos tecidos tumorais apresentaram um tempo de sobrevida menor do que os outros 
cães (Franzoni et al., 2019).

Quanto a intensidade do infiltrado inflamatório dos 33 neoplasmas analisados (Tabela 2), verificamos que a maioria dos neoplasmas apresentaram infiltrado inflamatório total e linfocitário menor que 800 células (baixa contagem) independentemente do tipo tumoral, grau ou tamanho. Não havia informação de grau de um neoplasma, e de tamanho de 4 neoplasmas, por não atender aos critérios de classificação.

Neste estudo, 66,7\% dos carcinomas mamários das gatas apresentou infiltrado linfocitário de baixa contagem, este dado se difere do encontrado em um estudo em cadelas, onde o infiltrado linfocitário intenso ( $\geq 800)$ predominou nos carcinomas mamários de cadelas (Lopes-Neto et al., 2017).

Tabela 2 - Avaliação da intensidade do infiltrado total e linfocitário dos carcinomas mamários de gatas.

\begin{tabular}{|c|c|c|c|c|c|c|c|c|}
\hline & & \multicolumn{3}{|c|}{ Infiltrado inflamatório } & \multicolumn{4}{|c|}{ Infiltrado linfocitário } \\
\hline & Parâmetros & $<800$ & $>800$ & $\mathrm{P}$ & $<800$ & $>800$ & $\mathrm{P}$ & $\mathrm{N}$ \\
\hline \multirow{4}{*}{$\begin{array}{l}\text { Tipo } \\
\text { Histológico }\end{array}$} & Carcinoma sólido & $7(58,3 \%)$ & $5(41,7 \%)$ & - & $7(58,3 \%)$ & $5(41,7 \%)$ & - & 12 \\
\hline & Carcinoma tubular & $9(69,2 \%)$ & $4(30,8 \%)$ & 0,571 & $10(76,9 \%)$ & $3(23,1 \%)$ & 0,319 & 13 \\
\hline & Carcinoma cribriforme & $5(62,5 \%)$ & $3(37,5 \%)$ & 0,852 & $5(62,5 \%)$ & $3(37,5 \%)$ & 0,852 & 8 \\
\hline & & $21(63,6 \%)$ & $12(36,4 \%)$ & & $22(66,7 \%)$ & $11(33,3 \%)$ & & 33 \\
\hline \multirow{4}{*}{$\begin{array}{l}\text { Grau } \\
\text { histológico }\end{array}$} & Grau III & $12(57,1 \%)$ & $9(42,9 \%)$ & - & $13(61,9 \%)$ & $8(38,1 \%)$ & - & 21 \\
\hline & Grau II & $4(57,1 \%)$ & $3(42,9 \%)$ & 1,0 & $4(57,1 \%)$ & $3(42,9 \%)$ & 0,823 & 7 \\
\hline & Grau I & $4(100 \%)$ & - & 0,102 & $4(100 \%)$ & - & 0,134 & 4 \\
\hline & & $20(62,5 \%)$ & $12(37,5 \%)$ & & $21(65,6 \%)$ & $11(34,4 \%)$ & & 32 \\
\hline \multirow[t]{4}{*}{ Tamanho } & $\mathrm{T} 3:>3 \mathrm{~cm}$ & $5(100,0 \%)$ & - & - & $5(100,0 \%)$ & - & - & 5 \\
\hline & $\mathrm{T} 2: 2-3 \mathrm{~cm}$ & $4(80 \%)$ & $1(20 \%)$ & 0,292 & $4(80 \%)$ & $1(20 \%)$ & 0,292 & 5 \\
\hline & $\mathrm{T} 1:<2 \mathrm{~cm}$ & $10(52,6 \%)$ & $9(47,4 \%)$ & 0,052 & $11(57,9 \%)$ & $8(42,1 \%)$ & 0,076 & 19 \\
\hline & & $19(65,5 \%)$ & $10(34,5 \%)$ & & $20(69 \%)$ & $9(31 \%)$ & & 29 \\
\hline
\end{tabular}

Fonte: Autores.

Um estudo conduzido por Dagher et al., (2019) em gatas demonstrou inflamação histiolinfocitária e plasmocítica moderada a grave em 51,4\% dos neoplasmas mamários, além disso, embora a avaliação tenha sido qualitativa, Dagher et al., (2019) demonstrou uma piora na sobrevida nas gatas com infiltrado moderado a grave. Em caninos o infiltrado linfocitário intenso foi associado a maior agressividade em carcinomas mamários, uma vez que se observou maior infiltrado de linfócitos em tumores com alto grau histológico, invasão de vasos linfáticos e necrose (Kim et al., 2013). Sugere-se que em cães um maior número de linfócitos tumorais possa ser necessário para o comportamento maligno (Lopes-Neto et al., 2017).

Um estudo conduzido em felinos relacionou a presença de infiltrado inflamatório crônico a uma piora no prognóstico, pois o infiltrado inflamatório crônico foi associado à necrose em carcinomas mamários (Weijer \& Hart, 1983). Porém, estudos adicionais que relacionam o infiltrado inflamatório e linfocitário com fatores prognósticos em felinos não são conhecidos.

Foram analisados 28 carcinomas mamários de felinos quanto a marcação de CD79a. Houve marcação positiva para CD79a em linfócitos e plasmócitos em 85,7\% dos neoplasmas (Figura 3), não havendo marcação em apenas 14,3\% dos casos analisados. 
Figura 3 - Fotomicrografia de imunoistoquímica em carcinoma mamário de gata utilizando anticorpo anti-CD79a. A) Marcação de linfócitos B no infiltrado inflamatório peritumoral. B) Marcação de linfócitos B em áreas de invasão tumoral. Notar células epiteliais neoplasias invasivas (*).

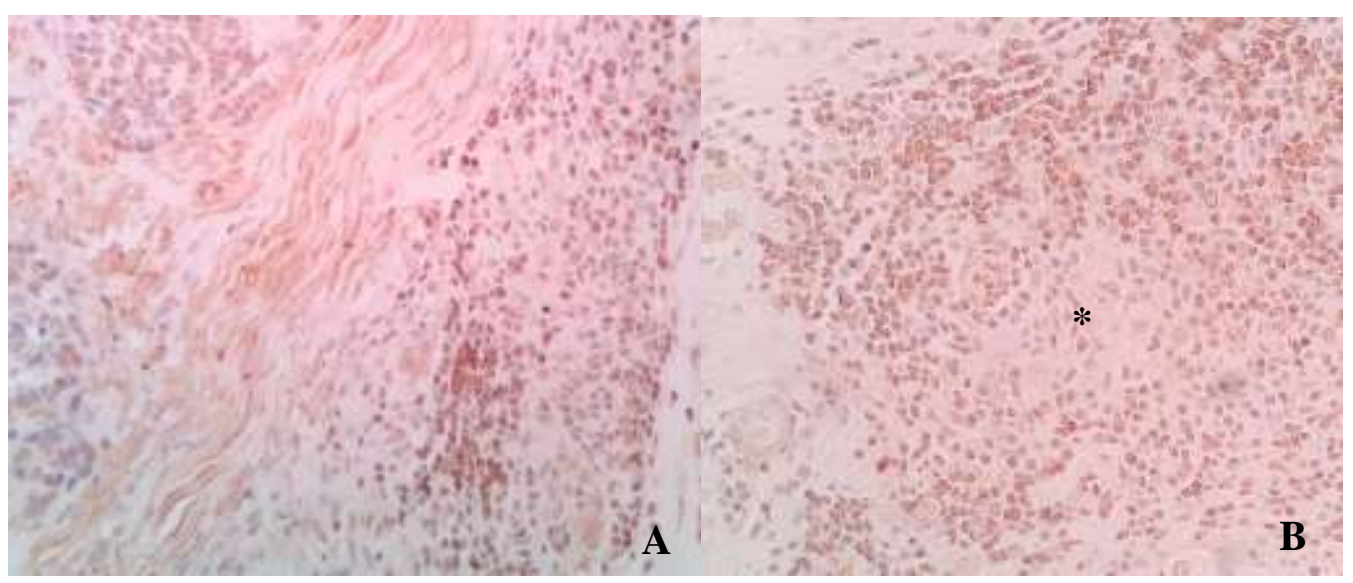

Fonte: Autores.

A maioria dos carcinomas apresentou áreas multifocais de infiltrado linfocitário, e as células CD79a+ eram encontradas distribuídas de forma difusa nos focos de infiltrado linfocitário, independentemente do tipo tumoral e grau histológico (Tabela 3), demostrando que os linfócitos B e plasmócitos são componentes integrantes do infiltrado inflamatório tumoral. Esses dados são semelhantes aos encontrados em um estudo de tumor de mama humano, onde o padrão predominante foi o difuso (Mahmoud et al., 2012). Houve um maior número de agregados em carcinomas sólidos em relação aos carcinomas tubulares e cribriformes, assim como nos neoplasmas de grau II e III.

Tabela 3 - Características dos carcinomas mamários quanto a densidade de marcação de CD79a.

\begin{tabular}{|c|c|c|c|c|c|c|}
\hline \multirow[b]{2}{*}{ Características } & \multicolumn{6}{|c|}{ Categorias de linfócitos B + Plasmócitos } \\
\hline & & Ausente & Difuso & Agregado & $\mathrm{N}$ & $\mathrm{P}$ \\
\hline Carcinoma tubular & & $3(27,3 \%)$ & $7(63,6 \%)$ & $1(9,1 \%)$ & 11 & 0,251 \\
\hline Carcinoma cribriforme & & $1(14,3 \%)$ & $5(71,4 \%)$ & $1(14,3 \%)$ & 7 & \\
\hline Carcinoma sólido & & - & $6(60 \%)$ & $4(40 \%)$ & 10 & \\
\hline Grau I & & - & $3(100 \%)$ & - & 3 & 0,414 \\
\hline Grau II & & $1(14,4 \%)$ & $3(42,8 \%)$ & $3(42,8 \%)$ & 7 & \\
\hline Grau III & & $3(16,7 \%)$ & $12(66,6 \%)$ & $3(16,7 \%)$ & 18 & \\
\hline \multirow[t]{2}{*}{ Invasão linfática } & Sim & $3(25 \%)$ & $9(75 \%)$ & - & 12 & $0,038^{*}$ \\
\hline & Não & $1(6,2 \%)$ & $9(56,3 \%)$ & $6(37,5 \%)$ & 16 & \\
\hline \multirow[t]{2}{*}{ Invasão tecidual } & Sim & $2(10 \%)$ & $14(70 \%)$ & $4(20 \%)$ & 20 & 0,516 \\
\hline & Não & $2(25 \%)$ & $4(50 \%)$ & $2(25 \%)$ & 8 & \\
\hline \multirow[t]{2}{*}{ Metástase linfonodo } & Sim & - & $4(57,1 \%)$ & $3(42,9 \%)$ & 7 & 0,257 \\
\hline & Não & - & $2(100 \%)$ & - & 2 & \\
\hline \multirow[t]{3}{*}{ Tamanho } & $<2 \mathrm{~cm}$ & $1(6,2 \%)$ & $10(62,5 \%)$ & $5(31,3 \%)$ & 16 & 0,137 \\
\hline & $2-3 \mathrm{~cm}$ & - & $3(100 \%)$ & - & 3 & \\
\hline & $>3 \mathrm{~cm}$ & $2(40 \%)$ & $3(60 \%)$ & - & 5 & \\
\hline
\end{tabular}

Fonte: Autores.

$\mathrm{Na}$ análise univariada, as categorias de imunomarcação (ausente, difuso e folicular/agregado) das células CD79a+ e os parâmetros histológicos avaliados (tipo histológico, grau, tamanho, invasão local, invasão linfovascular e metástase em linfonodo) revelaram diferença significativa para invasão linfática ( $\mathrm{P}=0,038)$.

A média geral das células imunomarcadas nos carcinomas foi de $50 \pm 70$, estes resultados são semelhantes aos encontrado em cães. As células B consistem no segundo linfócito infiltrador de tumor mais abundante (Yuen et al., 2016). Os 
resultados das médias de contagem de células CD79a quanto aos parâmetros analisados estão apresentadas na Tabela 4.

Tabela 4 - Quantificação de células CD79a quanto aos parâmetros histológicos analisados nos neoplasmas mamários malignos em felinos.

\begin{tabular}{|c|c|c|c|c|}
\hline & & CD79a & & \\
\hline Parâmetros & & Média & $\mathrm{N}$ & P\# \\
\hline Carcinoma tubular (a) & & $39,50 \pm 62,88$ & 11 & $(a-b)=0,121$ \\
\hline Carcinoma sólido (b) & & $76,54 \pm 92,98$ & 10 & $(b-c)=0,770$ \\
\hline Carcinoma cribrifirme (c) & & $28,58 \pm 17,10$ & 7 & $(a-c)=0,317$ \\
\hline Grau I & & $65,23 \pm 68,62$ & 3 & $(\mathrm{I}-\mathrm{II})=0,732$ \\
\hline Grau II & & $69,36 \pm 91,95$ & 7 & $(\mathrm{I}-\mathrm{III})=0,314$ \\
\hline Grau III & & $39,93 \pm 61,97$ & 18 & $(\mathrm{II}-\mathrm{III})=0,770$ \\
\hline Tamanho <2cm (d) & & $56,68 \pm 67,16$ & 16 & $(\mathrm{~d}-\mathrm{f})=0,045^{*}$ \\
\hline Tamanho $2-3 \mathrm{~cm}(\mathrm{e})$ & & $19,93 \pm 8,63$ & 3 & $(\mathrm{~d}-\mathrm{e})=0,5$ \\
\hline Tamanho $>3 \mathrm{~cm}(\mathrm{f})$ & & $12,5 \pm 17,15$ & 5 & $(e-f)=0,29$ \\
\hline \multirow[t]{2}{*}{ Invasão linfática } & Sim & $22,05 \pm 26,88$ & 12 & $0,039 *$ \\
\hline & Não & $70,96 \pm 84,15$ & 16 & \\
\hline \multirow[t]{2}{*}{ Invasão tecidual } & Sim & $56,41 \pm 79,26$ & 20 & 0,839 \\
\hline & Não & $33,97 \pm 34,54$ & 8 & \\
\hline \multirow[t]{2}{*}{ Metástase linfonodo } & Sim & $59,16 \pm 91,05$ & 7 & 0,079 \\
\hline & Não & $10,95 \pm 3,74$ & 2 & \\
\hline
\end{tabular}

Fonte: Autores.

Houve diferença significativa quanto ao tamanho tumoral, onde o número de células CD79a+ foi significativamente maior em carcinomas menores que $2 \mathrm{~cm}$ quando comparado aos tumores maiores que $3 \mathrm{~cm}(\mathrm{P}=0,045)$ e houve correlação significativa entre tamanho e média de células $C D 79 a$ positivas $(\mathrm{P}=0,045)$. As médias de células CD79a+ foi significativamente maior nos neoplasmas sem invasão linfática em comparação aos com invasão $(\mathrm{P}=0,039)$. Sugere-se que um maior número de células CD79 pode estar relacionado a um melhor prognostico em gatas, pois os valores maiores de células CD79 foram maiores em tumores pequenos e sem invasão linfática. Sabe-se que a invasão linfática e o tamanho são importantes fatores prognósticos para neoplasmas mamários de gatas e cadelas (Cassali et al., 2018, 2020). Ainda, neste estudo os neoplasmas T1 tiveram média células CD79 proporcionalmente maior em relação a número de linfócitos, quando comparado com os neoplasmas $\mathrm{T} 3$.

O microambiente tumoral é um fator chave na determinação das propriedades do potencial invasivo e metastático do tumor e parece também ser heterogêneo (Tashireva et al., 2015; Lopes-Neto et al., 2017). Em humanos, o microambiente tumoral na maioria dos neoplasmas sólidos é infiltrado por células T, macrófagos, fibroblastos e células dendríticas (Tashireva et al., 2015). As células inflamatórias no microambiente tumoral podem estar envolvidas na promoção ou na supressão do crescimento neoplásico. A interação entre células tumorais e outras células presentes no microambiente tumoral pode ser decisivo para o crescimento e a metástase. Isso pode ser potencializado pelo infiltrado inflamatório no microambiente tumoral através do remodelamento de tecidos e liberação de proteases, citocinas e fatores de crescimento (Salgado et al., 2015; Carvalho et al., 2016). Na análise morfológica das amostras em coloração de rotina, verificou-se neste estudo que a maioria das células inflamatórias no microambiente tumoral dos carcinomas mamários das gatas eram linfócitos e células CD79a positivas fazem parte destas células linfocitárias.

Neste estudo a presença de células B e plasmócitos (CD79a positivas) nos tumores sem invasão linfática pode corresponder a um possível efeito protetor, ou ainda, estas células podem estar presentes em maior número na fase inicial do desenvolvimento tumoral, uma vez que a média de células CD79 positivas e contagem de linfócitos na análise morfológica do infiltrado inflamatório tumoral foi maior nos tumores T1. Ainda, verificou-se que nos carcinomas de grau III a média de 
linfócitos foi maior em relação aos carcinomas de grau I, e inversamente, a média de células CD79 foi menor em tumores grau III em relação aos neoplasmas de grau I, portanto essas células podem estar associadas a tumores de menor malignidade como ocorre em cães, onde a presença de linfócitos B e plasmócitos estava associados a tumores mamários menos agressivos (de Souza et al., 2018).

\section{Considerações Finais}

Através do presente estudo concluiu-se que as células linfocitárias representam uma parcela significativa infiltrado inflamatório tumoral, sendo a população celular predominante. Estas células podem ter um potencial fator prognóstico nos carcinomas mamários de gatas. Este é o primeiro estudo a quantificar as células inflamatórias do microambiente tumoral em carcinomas mamários de gatas, e revela resultados iniciais promissores. As células CD79a positivas compõem uma porção significativa da população linfocitária do microambiente tumoral e fazem parte da resposta inflamatória associada ao tumor nos carcinomas mamários felinos. Estas células foram encontradas de forma difusa de modo geral em todos os neoplasmas, e podem estar relacionados ao comportamento biológico tumoral como tamanho e invasão linfática. Este estudo fornece dados preliminares para a caracterização de possíveis marcadores imunológicos e prognósticos para carcinomas mamários de felinos e como tal pode ser explorada em estudos futuros.

\section{Agradecimentos}

O presente trabalho foi realizado com apoio da Coordenação de Aperfeiçoamento de Pessoal de Nível Superior Brasil (CAPES) - Código de Financiamento 001.

\section{Referências}

Borrego, J. F., Cartagena, J. C., \& Engel, J. (2009). Treatment of feline mammary tumours using chemotherapy, surgery and a COX-2 inhibitor drug (meloxicam): A retrospective study of 23 cases (2002-2007). Veterinary and Comparative Oncology, 7(4), 213-221. 10.1111/j.1476-5829.2009.00194.x.

Campos, C. B., Damasceno, K. A., Gamba, C. O., Ribeiro, A. M., Machado, C. J., Lavalle, G. E., \& Cassali, G. D. (2016). Evaluation of prognostic factors and survival rates in malignant feline mammary gland neoplasms. Journal of Feline Medicine and Surgery, 18(12), 1003-1012. $10.1177 / 1098612 X 15610367$.

Carvalho, M. I., Pires, I., Prada, J., \& Queiroga, F. L. (2011). T-lymphocytic infiltrate in canine mammary tumours: Clinic and prognostic implications. In Vivo, 25(6), 963-969.

Carvalho, M. I., Silva-Carvalho, R., Pires, I., Prada, J., Bianchini, R., Jensen-Jarolim, E., \& Queiroga, F. L. (2016). A Comparative Approach of TumorAssociated Inflammation in Mammary Cancer between Humans and Dogs. BioMed Research International, 2016. 10.1155/2016/4917387.

Cassali, G. D., Campos, C. B., Bertagnolli, A. C., Lima, A. E., Lavalle, G. E., Damasceno, K. A., Nardi, A. B. de;, Cogliati, B., Costa, F. V. A. da;, Sobral, R., Di Santis, G. W., Fernandes, C. G., Ferreira, E., Salgado, B. S., Vieira Filho, C. H. C., Silva, D. N., Martins Filho, E. F., Teixeira, S. V., Nunes, F. C., \& Nakagaki, K. Y. R. (2018). Consensus for the diagnosis, prognosis and treatment of feline mammary tumors. Brazilian Journal of Veterinary Research and Animal Science, 55(2), 1-17. 10.11606/issn.1678-4456.bjvras.2018.135084.

Cassali, G. D., Jark, P., Gamba, C., Damasceno, K., Estrela-Lima, A., Nardi, A. B., Ferreira, E., Horta, R., Firmo, B., Sueiro, F., Rodrigues, L., \& Nakagaki, K. (2020). Consensus Regarding the Diagnosis, Prognosis and Treatment of Canine and Feline Mammary Tumors - 2019. Brazilian Journal of Veterinary Pathology, 13(3), 555-574. 10.24070/bjvp.1983-0246.v13i3p555-574.

Dagher, E., Abadie, J., Loussouarn, D., Campone, M., \& Nguyen, F. (2019). Feline Invasive Mammary Carcinomas: Prognostic Value of Histological Grading. Veterinary Pathology, 56(5), 660-670. 10.1177/0300985819846870.

De Souza, T. A., de Campos, C. B., De Biasi Bassani Gonçalves, A., Nunes, F. C., Monteiro, L. N., de Oliveira Vasconcelos, R., \& Cassali, G. D. (2018). Relationship between the inflammatory tumor microenvironment and different histologic types of canine mammary tumors. Research in Veterinary Science, 119(October 2017), 209-214. 10.1016/j.rvsc.2018.06.012.

Elston, C. W., \& Ellis, I. O. (1991). Pathological prognostic factors in breast cancer: experience from a large study with long-term follow-up. Histopathology, 19, 403-410. 10.1111/j.1365-2559.1991.tb00229.x.

Estrela-Lima, A., Araújo, M. S. S., Costa-Neto, J. M., Teixeira-Carvalho, A., Barrouin-Melo, S. M., Cardoso, S. V., Martins-Filho, O. A., Serakides, R., \& Cassali, G. D. (2010). Immunophenotypic features of tumor infiltrating lymphocytes from mammary carcinomas in female dogs associated with prognostic factors and survival rates. BMC Cancer, 10. 10.1186/1471-2407-10-256. 
Franzoni, M. S., Brandi, A., de Oliveira Matos Prado, J. K., Elias, F., Dalmolin, F., de Faria Lainetti, P., Prado, M. C. M., Leis-Filho, A. F., \& Fonseca-Alves, C. E. (2019). Tumor-infiltrating CD4+ and CD8+ lymphocytes and macrophages are associated with prognostic factors in triple-negative canine mammary complex type carcinoma. Research in Veterinary Science, 126(May), 29-36. 10.1016/j.rvsc.2019.08.021.

Goldschmidt, M. H., Peña, L., \& Zappulli, V. (2017). Tumors of the Mammary Gland. In Meuten D.J. (Ed.), Tumors in Domestic Animals. (5th ed.), 723-765. John Wiley \& Sons Inc.

Kim, J. H., Yu, C. H., Yhee, J. Y., Im, K. S., \& Sur, J. H. (2010). Lymphocyte infiltration, expression of interleukin (IL) -1, IL-6 and expression of mutated breast cancer susceptibility gene-1 correlate with malignancy of canine mammary tumours. Journal of Comparative Pathology, 142(2-3), 177-186. 10.1016/j.jcpa.2009.10.023.

Kim, J. H., Chon, S. K., Im, K. S., Kim, N. H., \& Sur, J. H. (2013). Correlation of tumor-infiltrating lymphocytes to histopathological features and molecular phenotypes in canine mammary carcinoma: A morphologic and immunohistochemical morphometric study. Canadian Journal of Veterinary Research, 77(2), $142-149$.

Knief, J., Reddemann, K., Petrova, E., Herhahn, T., Wellner, U., \& Thorns, C. (2016). High density of tumor-infiltrating b-lymphocytes and plasma cells signifies prolonged overall survival in adenocarcinoma of the esophagogastric junction. Anticancer Research, 36(10), 5339-5345. 10.21873/anticanres.11107.

Lopes-Neto, B. E., Caroline, S., Souza, B., Bouty, L. M., Jonas, G., Santos, L., \& Oliveira, E. S. (2017). CD4+, CD8+, FoxP3+ and HSP60+ Expressions in Cellular Infiltrate of Canine Mammary Carcinoma in Mixed Tumor. Acta Scientiae Veterinariae, 55(85), 1-8. 10.22456/1679-9216.80758.

Macchetti, A. H., Marana, H. R. C., Silva, J. S., De Andrade, J. M., Ribeiro-Silva, A., \& Bighetti, S. (2006). Tumor-infiltrating CD4+ T lymphocytes in early breast cancer reflect lymph node involvement. Clinics, 61(3), 203-208. 10.1590/S1807-59322006000300004.

Mahmoud, S. M. A., Lee, A. H. S., Paish, E. C., MacMillan, R. D., Ellis, I. O., \& Green, A. R. (2012). The prognostic significance of B ly mphocytes in invasive carcinoma of the breast. Breast Cancer Research and Treatment, 132(2), 545-553. 10.1007/s10549-011-1620-1.

O’Neill, K., Guth, A., Biller, B., Elmslie, R., \& Dow, S. (2009). Changes in regulatory T cells in dogs with cancer and associations with tumor type. Journal of Veterinary Internal Medicine, 23(4), 875-881. 10.1111/j.1939-1676.2009.0333.x.

Pérez, J., Day, M. J., Martín, M. P., Gonzalez, S., \& Mozos, E. (1999). Immunohistochemical study of the inflammatory infiltrate associated with feline cutaneous squamous cell carcinomas and precancerous lesions (actinic keratosis). Veterinary Immunology and Immunopathology, 1;69(1):33-46. $10.1016 / \mathrm{s} 0165-2427(99) 00032-\mathrm{x}$.

Salgado, R., Denkert, C., Demaria, S., Sirtaine, N., Klauschen, F., Pruneri, G., Wienert, S., Van den Eynden, G., Baehner, F. L., Penault-Llorca, F., Perez, E. A., Thompson, E. A., Symmans, W. F., Richardson, A. L., Brock, J., Criscitiello, C., Bailey, H., Ignatiadis, M., Floris, G., ... Loi, S. (2015). The evaluation of tumor-infiltrating lymphocytes (TILS) in breast cancer: Recommendations by an International TILS Working Group 2014. Annals of Oncology, 26(2), 259271. 10.1093/annonc/mdu450.

Seixas, F., Antunes, D., \& Pires, M. A. (2018). Immunohistochemical Analysis of T Lymphocytes (CD3 +) in Feline Mammary Lesions. Journal of Comparative Pathology, 158, 126. 10.1016/j.jcpa.2017.10.096.

Seixas, F., Palmeira, C., Pires, M. A., Bento, M. J., \& Lopes, C. (2011). Grade is an independent prognostic factor for feline mammary carcinomas: A clinicopathological and survival analysis. Veterinary Journal, 187(1), 65-71. 10.1016/j.tvj1.2009.10.030.

Shen, M., Wang, J., \& Ren, X. (2018). New insights into tumor-infiltrating B lymphocytes in breast cancer: Clinical impacts and regulatory mechanisms. Frontiers in Immunology, 9, 1-8. 10.3389/fimmu.2018.00470.

Tashireva, L., Denisov, E., Savelieva, O., Zavyalova, M., Kaigorodova, E., Slonimskaya, E., \& Perelmuter, V. (2015). A14: Heterogeneity in breast tumor microenvironment: A report from one case. European Journal of Cancer Supplements, 13(1), 61. 10.1016/j.ejcsup.2015.08.109.

Togni, M., Masuda, E. K., Kommers, G. D., Fighera, R. A., \& Irigoyen, L. F. (2013). Estudo retrospectivo de 207 casos de tumores mamários em gatas. Pesquisa Veterinária Brasileira, 33(3), 353-358. 10.1590/S0100-736X2013000300013.

Viste, J. R., Myers, S. L., Singh, B., \& Simko, E. (2002). Feline mammary adenocarcinoma: Tumor size as a prognostic indicator. Canadian Veterinary Journal, 43(1), 33-37. 10.1111/j.1751-0813.2002.tb11342.x.

Weijer, K., \& Hart, A. A. M. (1983). Prognostic Factors in Feline Mammary Carcinoma. JNCI: Journal of the National Cancer Institute, 70(4). $10.1093 /$ jnci/70.4.709.

Wouters, M. C. A., \& Nelson, B. H. (2018). Prognostic significance of tumor-infiltrating B cells and plasma cells in human cancer. Clinical Cancer Research, 24(24), 6125-6135. 10.1158/1078-0432.CCR-18-1481.

Yuen, G. J., Demissie, E., \& Pillai, S. (2016). B Lymphocytes and Cancer: A Love-Hate Relationship. Trends in Cancer, 2(12), 747-757. 10.1016/j.trecan.2016.10.010.

Zappulli, V., Rasotto, R., Caliari, D., Mainenti, M., Peña, L., Goldschmidt, M. H., \& Kiupel, M. (2015). Prognostic Evaluation of Feline Mammary Carcinomas: A Review of the Literature. Veterinary Pathology, 52(1), 46-60. 10.1177/0300985814528221. 